PACS: 02.20.-a, 78.55.-m, 78.40.Fy

\title{
Application of the method of projective representations to the analysis of exciton-phonon transitions in enantiomorphous tetragonal crystals $\mathrm{ZnP}_{2}$ and $\mathrm{CdP}_{2}$
}

\author{
S.V. Koryakov, V.O. Gubanov, M.M. Biliy, O.V. Slobodyanyuk, Z.Z. Yanchuk \\ Taras Shevchenko Kyiv National University, Department of Physics \\ 2, prospect Academician Glushkov, 03022 Kyiv, Ukraine \\ Phone: +38 (067) 465-64-18 \\ E-mail: sergiy_koryakov@univ.kiev.ua
}

\begin{abstract}
The projective representations of the wavevector group were applied to the analysis of exciton-phonon transitions in enantiomorphous tetragonal crystals $\mathrm{ZnP}_{2}$ and $\mathrm{CdP}_{2}$. Selection rules for indirect transitions were derived. Using $\vec{k} \vec{p}$-method, the different points in the Brillouin zone were examined to identify zero slopes and extremums of the dispersion curves $E(\vec{k})$. Photoluminescence and edge absorption polarized spectra were interpreted taking into account the results of above theoretical analysis.
\end{abstract}

Keywords: projective representations, exciton-phonon transitions, $\alpha-\mathrm{ZnP}_{2}, \mathrm{CdP}_{2}$.

Manuscript received 12.11.04; accepted for publication 18.05.05.

Symmetry analysis helps to predict some basic features of electronic transitions that appear as bands in optical spectra. The transition probability is defined by the product of the squared transition matrix element and combined density of states. Symmetry analysis gives selection rules, i.e., it shows what matrix elements are non-zero. The high density of states is expected in the vicinity of points with zero slope and/or extremums of the energy dispersion curves $E(\vec{k})$.

In this paper, we represent the method of selection rule calculations for exciton-phonon transitions in the tetragonal enantiomorphous crystals $\alpha-\mathrm{ZnP}_{2}$ and $\mathrm{CdP}_{2}$. These crystals belong to $P 4_{1} 2_{1} 2$ or $P 4_{3} 2_{1} 2$ space symmetry groups. Earlier projective representations were used for analysis of one- and two-phonon Raman spectra of the same crystals $[1,2]$.

The tetragonal crystals of zinc and cadmium diphosphides are indirect semiconductors, so it is necessary to examine interband transitions between different points of the Brillouin zone (Fig. 1). Here we examine the high symmetry points $\Gamma\left(\vec{k}_{\Gamma}=0\right)$, $\mathrm{Z}\left(\vec{k}_{\mathrm{Z}}=-\frac{\vec{b}_{1}}{2}\right), \mathrm{A}\left(\vec{k}_{\mathrm{A}}=-\frac{\vec{b}_{1}}{2}-\frac{\vec{b}_{2}}{2}-\frac{\vec{b}_{3}}{2}\right), \mathrm{M}\left(\vec{k}_{\mathrm{M}}=-\frac{\vec{b}_{2}}{2}-\frac{\vec{b}_{3}}{2}\right)$, $\mathrm{X}\left(\vec{k}_{\mathrm{X}}=-\frac{\vec{b}_{2}}{2}\right)$ and $\mathrm{X}^{\prime}\left(\vec{k}_{\mathrm{X}^{\prime}}=-\frac{\vec{b}_{3}}{2}\right), \mathrm{R}\left(\vec{k}_{\mathrm{R}}=-\frac{\vec{b}_{1}}{2}-\frac{\vec{b}_{2}}{2}\right)$ and $\mathrm{R}^{\prime}\left(\vec{k}_{\mathrm{R}^{\prime}}=-\frac{\vec{b}_{1}}{2}-\frac{\vec{b}_{3}}{2}\right)$. Points $\Gamma, \mathrm{Z}, \mathrm{A}$, and M possess full symmetry of the point group 422 as well as $\mathrm{X}, \mathrm{X}^{\prime}, \mathrm{R}$, and $\mathrm{R}^{\prime}$ ones belong to the point group 222 . To calculate selection rules and to ascertain whether the mentioned points are those of band extremums, it is necessary to know irreducible representations of wavevector groups. The irreducible representations of the wavevector groups can be found using the method of projective representations $[1,3]$.

To derive the selection rules for exciton-phonon transitions, we need to know representations both for phonon and electron states. The electron states are transformed according to the representations $\Gamma_{6}, \Gamma_{7}$, $\left(\mathrm{Z}_{1}+\mathrm{Z}_{2}\right), \quad\left(\mathrm{Z}_{3}+\mathrm{Z}_{4}\right), \quad \mathrm{Z}_{5}, \quad\left(\mathrm{~A}_{1}+\mathrm{A}_{3}\right), \quad\left(\mathrm{A}_{2}+\mathrm{A}_{4}\right)$, $\left(\left(A_{5}\right)\right) \cdot\left(M_{6}+M_{7}\right), \quad\left(X_{1}+X_{3}\right), \quad\left(X_{2}+X_{4}\right), \quad\left(X_{1}^{\prime}+X_{4}^{\prime}\right)$, $\left(\mathrm{X}_{2}^{\prime}+\mathrm{X}_{3}^{\prime}\right),\left(\left(\mathrm{R}_{5}\right)\right)$, and $\left(\left(\mathrm{R}_{5}^{\prime}\right)\right)$. The phonon states are transformed according to the representations $\Gamma_{1}-\Gamma_{5}, Z_{6}$, $\mathrm{Z}_{7},\left(\mathrm{~A}_{6}+\mathrm{A}_{7}\right),\left(\mathrm{M}_{1}+\mathrm{M}_{4}\right),\left(\mathrm{M}_{2}+\mathrm{M}_{3}\right), \mathrm{M}_{5}, \mathrm{X}_{5},\left(\mathrm{R}_{1}+\mathrm{R}_{4}\right)$, $\left(\mathrm{R}_{2}+\mathrm{R}_{3}\right)$, $\left(\mathrm{R}_{1}^{\prime}+\mathrm{R}_{3}^{\prime}\right)$, and $\left(\mathrm{R}_{2}^{\prime}+\mathrm{R}_{4}^{\prime}\right)$. Parentheses are for merging of complex conjugated representations. Double parentheses are for duplication of two-dimensional representations. The merging and duplication of representations are caused by the invariance with respect to the time inversion and governed by the Herring criterion [4]. The projective representations for $\Lambda, \mathrm{S}, \mathrm{V}$, $\Sigma, \Delta, \mathrm{W}$, and $\mathrm{U}$ points depend on the wavevector [3]. Their evolution in certain direction can be pictured graphically as shown in Fig. 2 for $\Gamma-\Sigma-M$. 


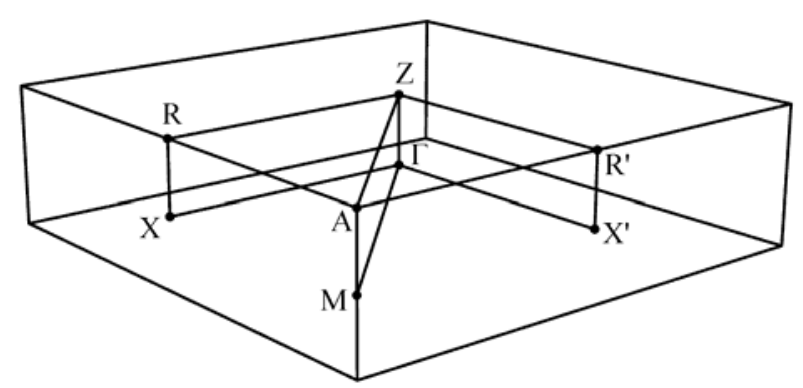

Fig. 1. Schematic drawing of the Brillouin zone that corresponds to tetragonal crystals of space symmetry groups $P 4_{1} 2_{1} 2$ and $P 4_{3} 2_{1} 2$.

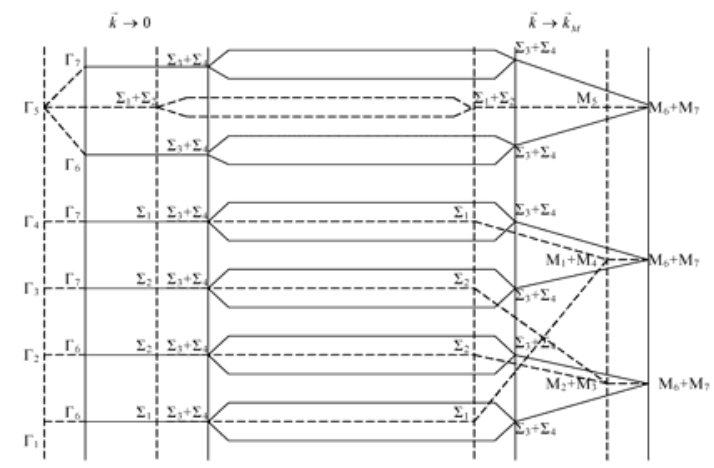

Fig. 2. Evolution of representations in $\Gamma-\mathrm{M}$ direction for groups $P 4_{1} 2_{1} 2$ (solid line - electron states, dashed line phonon states).

The selection rules for exciton-phonon transitions follow from the formula:

$N_{0}^{\mathrm{ex}-\mathrm{ph}}=\frac{1}{h} \sum_{g \in G_{\vec{k}}} \chi_{\vec{k}^{\prime} \mu}^{*}(g) \chi_{s}(g) \chi_{\kappa}(g) \chi_{\vec{k} v}(g) \chi_{\vec{q} \eta}(g)$,

where $\chi_{\vec{k}^{\prime} \mu}^{*}(g)$ and $\chi_{\overrightarrow{k v}}(g)$ are characters of representations of hole and electron states in valence and conduction bands, respectively; $\chi_{s}(g)$ is the character of representation of the dipole transition operator; $\chi_{\kappa}(g)$ is the character of the hole and electron moving relatively to their center of gravity, $\chi_{\vec{q} \eta}(g)$ is the character of the phonon state representation. For the allowed transition $N_{0}^{\mathrm{ex}-\mathrm{ph}} \neq 0$, and for the forbidden one $N_{0}^{\text {ex-ph }}=0$. If any external magnetic field is absent, the characters of merged representations have to be used (selection rules are shown in Table 1).

For the point $\vec{k}_{0}$ with zero slope of energy dispersion curves, the condition $d E\left(\vec{k}_{0}\right) / d k_{\alpha}=0$ should be fulfilled. In addition, in the point of extremum the components of inverse effective mass tensor $1 / m_{\alpha \beta}$
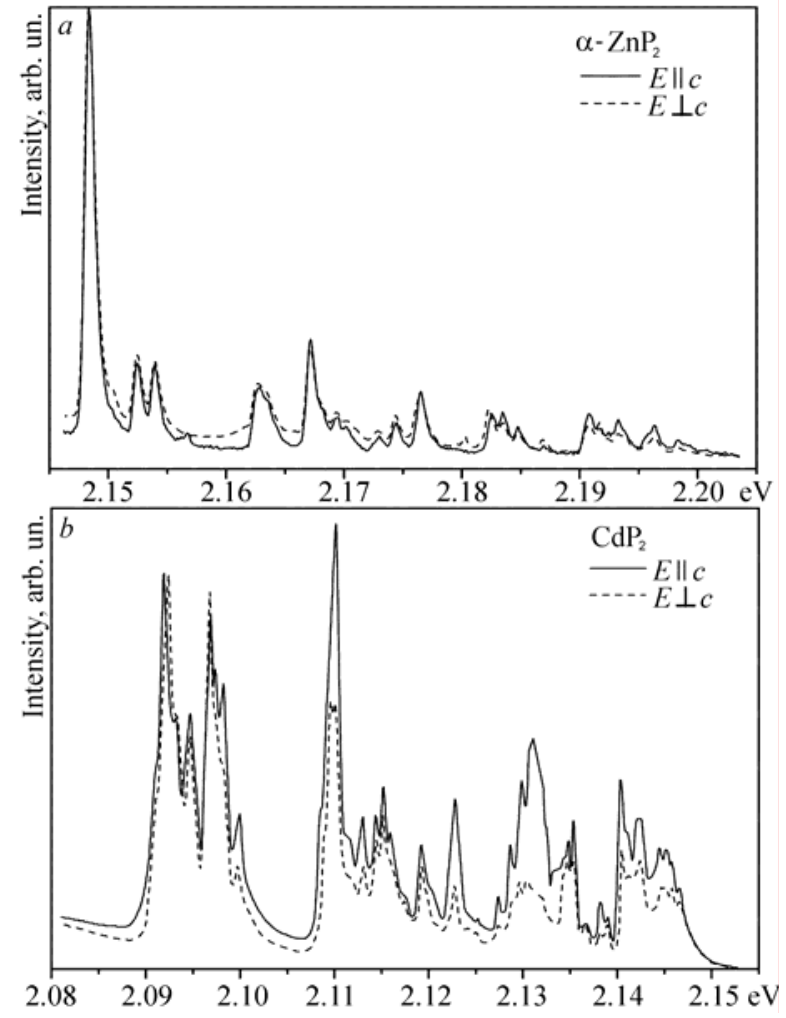

Fig. 3. Photoluminescence spectra of tetragonal crystals $\mathrm{ZnP}_{2}$ (a) and $\mathrm{CdP}_{2}$ (b).

should not be equal to zero. We have analyzed using the $\vec{k} \vec{p}$-method [4] whether examined points are the critical ones (results are summarized in Tables 2 and 3). From Table 3 , it is easy to see that there are non-zero components of the inverse effective mass tensor $\frac{1}{m_{\alpha \beta}}$ in every examined point of the Brillouin zone. It means that some dispersion curves of $E(\vec{k})$ may have the extremum in these points. But not all points are those of zero slope (see Table 2). There is no point in the Brillouin zone that has all zero components $d E\left(\vec{k}_{0}\right) / d k_{\alpha}$ for both electron and phonon states.

Photoluminescence and absorption polarized spectra of $\alpha-\mathrm{ZnP}_{2}$ and $\mathrm{CdP}_{2}$ (Figs 3, 4) were obtained at the temperature $1.7 \mathrm{~K}$ using a spectrometer DFS-24 with the resolution less than $1 \mathrm{~cm}^{-1}$ at $\lambda=550 \mathrm{~nm}$. Photoluminescence was excited by radiation of $\mathrm{Ar}^{+}$laser $(\lambda=514.5 \mathrm{~nm})$ with the output power $900 \mathrm{~mW}$. The main feature of photoluminescence spectra in both polarizations (Fig. 3) is the equal number of bands with approximately the same phonon energies. It means that all the observed interband transitions are allowed in both polarizations. 
Table 1. Selection rules for exciton-phonon transitions between different points of the Brillouin zone in tetragonal crystals $\mathrm{ZnP}_{2}$ and $\mathrm{CdP}_{2}$.

\begin{tabular}{|c|c|c|}
\hline $\begin{array}{l}\text { Type of } \\
\text { transition }\end{array}$ & Allowed transitions & Forbidden transitions \\
\hline$\Gamma-Z$ & $\begin{array}{r}\Gamma_{2} \subset \Gamma_{6} \times\left(Z_{1}+Z_{2}\right) \times Z_{7}, \Gamma_{6} \times\left(Z_{3}+Z_{4}\right) \times Z_{6}, \Gamma_{6} \times Z_{5} \times Z_{6}, \Gamma_{6} \times Z_{5} \times Z_{7}, \\
\Gamma_{7} \times\left(Z_{1}+Z_{2}\right) \times Z_{6}, \Gamma_{7} \times\left(Z_{3}+Z_{4}\right) \times Z_{7}, \Gamma_{7} \times Z_{5} \times Z_{6}, \Gamma_{7} \times Z_{5} \times Z_{7} \\
\Gamma_{5} \subset \Gamma_{6} \times\left(Z_{1}+Z_{2}\right) \times Z_{6}, \Gamma_{6} \times\left(Z_{1}+Z_{2}\right) \times Z_{7}, \Gamma_{6} \times\left(Z_{3}+Z_{4}\right) \times Z_{6}, \\
\Gamma_{6} \times\left(Z_{3}+Z_{4}\right) \times Z_{7}, \Gamma_{6} \times Z_{5} \times Z_{6}, \Gamma_{6} \times Z_{5} \times Z_{7}, \Gamma_{7} \times\left(Z_{1}+Z_{2}\right) \times Z_{6}, \\
\Gamma_{7} \times\left(Z_{1}+Z_{2}\right) \times Z_{7}, \Gamma_{7} \times\left(Z_{3}+Z_{4}\right) \times Z_{6}, \Gamma_{7} \times Z_{5} \times Z_{6}, \Gamma_{7} \times Z_{5} \times Z_{7}\end{array}$ & $\begin{array}{l}\Gamma_{2} \subset \Gamma_{6} \times\left(Z_{1}+Z_{2}\right) \times Z_{6}, \Gamma_{6} \times\left(Z_{3}+Z_{4}\right) \times Z_{7}, \\
\quad \Gamma_{7} \times\left(Z_{1}+Z_{2}\right) \times Z_{7}, \Gamma 7 \times\left(Z_{3}+Z_{4}\right) \times Z_{6} \\
\Gamma_{5} \subset 0\end{array}$ \\
\hline$\Gamma-\mathrm{A}$ & $\begin{array}{c}\Gamma_{2} \subset \Gamma_{6} \times\left(\mathrm{A}_{1}+\mathrm{A}_{2}\right) \times\left(\mathrm{A}_{6}+\mathrm{A}_{7}\right), \Gamma_{6} \times\left(\mathrm{A}_{3}+\mathrm{A}_{4}\right) \times\left(\mathrm{A}_{6}+\mathrm{A}_{7}\right), \\
\Gamma_{6} \times 2 \mathrm{~A}_{5} \times\left(\mathrm{A}_{6}+\mathrm{A}_{7}\right), \Gamma_{7} \times\left(\mathrm{A}_{1}+\mathrm{A}_{2}\right) \times\left(\mathrm{A}_{6}+\mathrm{A}_{7}\right), \\
\Gamma_{7} \times\left(\mathrm{A}_{3}+\mathrm{A}_{4}\right) \times\left(\mathrm{A}_{6}+\mathrm{A}_{7}\right), \Gamma_{7} \times 2 \mathrm{~A}_{5} \times\left(\mathrm{A}_{6}+\mathrm{A}_{7}\right) \\
\Gamma_{5} \subset \Gamma_{6} \times\left(\mathrm{A}_{1}+\mathrm{A}_{2}\right) \times\left(\mathrm{A}_{6}+\mathrm{A}_{7}\right), \Gamma_{6} \times\left(\mathrm{A}_{3}+\mathrm{A}_{4}\right) \times\left(\mathrm{A}_{6}+\mathrm{A}_{7}\right), \\
\Gamma_{6} \times 2 \mathrm{~A}_{5} \times\left(\mathrm{A}_{6}+\mathrm{A}_{7}\right), \Gamma_{7} \times\left(\mathrm{A}_{1}+\mathrm{A}_{2}\right) \times\left(\mathrm{A}_{6}+\mathrm{A}_{7}\right), \\
\Gamma_{7} \times\left(\mathrm{A}_{3}+\mathrm{A}_{4}\right) \times\left(\mathrm{A}_{6}+\mathrm{A}_{7}\right), \Gamma_{7} \times 2 \mathrm{~A}_{5} \times\left(\mathrm{A}_{6}+\mathrm{A}_{7}\right)\end{array}$ & $\begin{array}{l}\Gamma_{2} \subset 0 \\
\Gamma_{5} \subset 0\end{array}$ \\
\hline$\Gamma-\mathrm{M}$ & $\begin{aligned} \Gamma_{2} \subset \Gamma_{6} \times\left(M_{1}+M_{2}\right) \times\left(M_{6}+M_{7}\right), \Gamma_{6} \times\left(M_{3}+M_{4}\right) \times\left(M_{6}+M_{7}\right), & \Gamma_{6} \times M_{5} \times\left(M_{6}+M_{7}\right), \Gamma_{7} \times\left(M_{1}+M_{2}\right) \times\left(M_{6}+M_{7}\right), \\
& \Gamma_{7} \times\left(M_{3}+A_{4}\right) \times\left(M_{6}+M_{7}\right), \Gamma_{7} \times 2 M_{5} \times\left(M_{6}+M_{7}\right) \\
\Gamma_{5} \subset & \Gamma_{6} \times\left(M_{1}+M_{2}\right) \times\left(M_{6}+M_{7}\right), \Gamma_{6} \times\left(M_{3}+M_{4}\right) \times\left(M_{6}+M_{7}\right), \\
& \Gamma_{6} \times M_{5} \times\left(M_{6}+M_{7}\right), \Gamma_{7} \times\left(M_{1}+M_{2}\right) \times\left(M_{6}+M_{7}\right), \\
& \Gamma_{7} \times\left(M_{3}+A_{4}\right) \times\left(M_{6}+M_{7}\right), \Gamma_{7} \times 2 M_{5} \times\left(M_{6}+M_{7}\right)\end{aligned}$ & $\begin{array}{l}\Gamma_{2} \subset 0 \\
\Gamma_{5} \subset 0\end{array}$ \\
\hline$\Gamma-\mathrm{X}$ & $\begin{array}{r}\Gamma_{2} \subset \Gamma_{6} \times\left(\mathrm{X}_{1}+\mathrm{X}_{3}\right) \times \mathrm{X}_{5}, \\
\Gamma_{6} \times\left(\mathrm{X}_{2}+\mathrm{X}_{4}\right) \times \mathrm{X}_{5}, \\
\Gamma_{7} \times\left(\mathrm{X}_{1}+\mathrm{X}_{3}\right) \times \mathrm{X}_{5}, \\
\Gamma_{7} \times\left(\mathrm{X}_{2}+\mathrm{X}_{4}\right) \times \mathrm{X}_{5} \\
\Gamma_{5} \subset \Gamma_{6} \times\left(\mathrm{X}_{1}+\mathrm{X}_{3}\right) \times \mathrm{X}_{5}, \\
\Gamma_{6} \times\left(\mathrm{X}_{2}+\mathrm{X}_{4}\right) \times \mathrm{X}_{5}, \\
\Gamma_{7} \times\left(\mathrm{X}_{1}+\mathrm{X}_{3}\right) \times \mathrm{X}_{5}, \\
\Gamma_{7} \times\left(\mathrm{X}_{2}+\mathrm{X}_{4}\right) \times \mathrm{X}_{5}\end{array}$ & $\Gamma_{2} \subset 0$ \\
\hline$\Gamma-\mathrm{X}^{\prime}$ & $\begin{array}{r}\Gamma_{2} \subset \Gamma_{6} \times\left(X_{1}^{\prime}+X_{4}^{\prime}\right) \times X_{5}^{\prime}, \\
\Gamma_{6} \times\left(X_{2}^{\prime}+X_{3}^{\prime}\right) \times X_{5}^{\prime}, \\
\Gamma_{7} \times\left(X_{1}^{\prime}+X_{4}^{\prime}\right) \times X_{5}^{\prime}, \\
\Gamma_{7} \times\left(X_{2}^{\prime}+X_{3}^{\prime}\right) \times X_{5}^{\prime}, \\
\Gamma_{5} \subset \Gamma_{6} \times\left(X_{1}^{\prime}+X_{4}^{\prime}\right) \times X_{5}^{\prime}, \\
\Gamma_{6} \times\left(X_{2}^{\prime}+X_{3}^{\prime}\right) \times X_{5}^{\prime}, \\
\Gamma_{7} \times\left(X_{1}^{\prime}+X_{4}^{\prime}\right) \times X_{5}^{\prime}, \\
\Gamma_{7} \times\left(X_{2}^{\prime}+X_{3}^{\prime}\right) \times X_{5}^{\prime}\end{array}$ & $\Gamma_{2} \subset 0$ \\
\hline
\end{tabular}




\begin{tabular}{c|c|c}
\hline & $\Gamma_{2} \subset \Gamma_{6} \times\left(\left(\mathrm{R}_{5}\right)\right) \times\left(\mathrm{R}_{1}+\mathrm{R}_{4}\right)$, & $\Gamma_{2} \subset 0$ \\
& $\Gamma_{6} \times\left(\left(\mathrm{R}_{5}\right)\right) \times\left(\mathrm{R}_{2}+\mathrm{R}_{3}\right)$, & \\
$\Gamma_{7} \times\left(\left(\mathrm{R}_{5}\right)\right) \times\left(\mathrm{R}_{1}+\mathrm{R}_{4}\right)$, & \\
$\Gamma_{7} \times\left(\left(\mathrm{R}_{5}\right)\right) \times\left(\mathrm{R}_{2}+\mathrm{R}_{3}\right)$ & \\
& $\Gamma_{5} \subset \Gamma_{6} \times\left(\left(\mathrm{R}_{5}\right)\right) \times\left(\mathrm{R}_{1}+\mathrm{R}_{4}\right)$, & $\Gamma_{5} \subset 0$ \\
$\Gamma_{6} \times\left(\left(\mathrm{R}_{5}\right)\right) \times\left(\mathrm{R}_{2}+\mathrm{R}_{3}\right)$, & \\
$\Gamma_{7} \times\left(\left(\mathrm{R}_{5}\right)\right) \times\left(\mathrm{R}_{1}+\mathrm{R}_{4}\right)$, & \\
& $\Gamma_{7} \times\left(\left(\mathrm{R}_{5}\right)\right) \times\left(\mathrm{R}_{2}+\mathrm{R}_{3}\right)$ & \\
\hline & $\Gamma_{2} \subset \Gamma_{6} \times\left(\left(\mathrm{R}_{5}^{\prime}\right)\right) \times\left(\mathrm{R}_{1}^{\prime}+\mathrm{R}_{3}^{\prime}\right)$, & \\
& $\Gamma_{6} \times\left(\left(\mathrm{R}_{5}^{\prime}\right)\right) \times\left(\mathrm{R}_{2}^{\prime}+\mathrm{R}_{4}^{\prime}\right)$, & \\
$\Gamma_{7} \times\left(\left(\mathrm{R}_{5}^{\prime}\right)\right) \times\left(\mathrm{R}_{1}+\mathrm{R}_{3}^{\prime}\right)$, & \\
$\Gamma_{7} \times\left(\left(\mathrm{R}_{5}^{\prime}\right)\right) \times\left(\mathrm{R}_{2}^{\prime}+\mathrm{R}_{4}^{\prime}\right)$ & \\
& $\Gamma_{5} \subset \Gamma_{6} \times\left(\left(\mathrm{R}_{5}^{\prime}\right)\right) \times\left(\mathrm{R}_{1}^{\prime}+\mathrm{R}_{3}^{\prime}\right)$, & \\
& $\Gamma_{6} \times\left(\left(\mathrm{R}_{5}^{\prime}\right)\right) \times\left(\mathrm{R}_{2}^{\prime}+\mathrm{R}_{4}^{\prime}\right)$, & \\
& $\Gamma_{7} \times\left(\left(\mathrm{R}_{5}^{\prime}\right)\right) \times\left(\mathrm{R}_{1}^{\prime}+\mathrm{R}_{3}^{\prime}\right)$, & \\
& $\Gamma_{7} \times\left(\left(\mathrm{R}_{5}^{\prime}\right)\right) \times\left(\mathrm{R}_{2}^{\prime}+\mathrm{R}_{4}^{\prime}\right)$ & \\
\hline
\end{tabular}

Table 2. Number of components of momentum for different points in the Brillouin zone of crystals $\mathrm{ZnP}_{2}$ and $\mathrm{CdP}_{2}$.

\begin{tabular}{|c|c|c|c|}
\hline \multirow{2}{*}{ Representation } & \multicolumn{3}{|c|}{ Number of components $d E\left(\vec{k}_{0}\right) / d k_{\alpha} \neq 0$} \\
\hline & $\mid z$ & \multicolumn{2}{|r|}{$\perp z$} \\
\hline$\overline{\Gamma_{1}, \Gamma_{2}, \Gamma_{3}, \Gamma_{4}}$ & \multirow{2}{*}{$\begin{array}{l}0 \\
1\end{array}$} & \multicolumn{2}{|r|}{0} \\
\hline & & \multirow{2}{*}{\multicolumn{2}{|c|}{$\begin{array}{l}0 \\
1\end{array}$}} \\
\hline$\Gamma_{6}, \Gamma_{7}$ & $\begin{array}{l}1 \\
1\end{array}$ & & \\
\hline $\begin{array}{l}\left(Z_{1}+Z_{2}\right),\left(Z_{3}+Z_{4}\right) \\
Z_{5} \\
Z_{6}, Z_{7}\end{array}$ & $\begin{array}{c}2 \text { diagonal; } 2 \text { non-diago } \\
1 \\
0\end{array}$ & & $\begin{array}{c}0 \text { diagonal; } 0 \text { non-diagonal } \\
0\end{array}$ \\
\hline $\begin{array}{l}\left(\mathrm{M}_{1}+\mathrm{M}_{4}\right),\left(\mathrm{M}_{2}+\mathrm{M}_{3}\right) \\
\mathrm{M}_{5} \\
\left(\mathrm{M}_{6}+\mathrm{M}_{7}\right)\end{array}$ & $\begin{array}{c}0 \text { diagonal; } 0 \text { non-diagonal } \\
1 \\
2 \text { diagonal; } 1 \text { non-diagonal }\end{array}$ & \multicolumn{2}{|c|}{$\begin{array}{c}0 \text { diagonal; } 0 \text { non-diagonal } \\
0 \\
2 \text { diagonal; } 3 \text { non-diagonal }\end{array}$} \\
\hline$\left(\mathrm{M}_{6}+\mathrm{M}_{7}\right)$ & \multicolumn{3}{|c|}{ in $\Gamma-A$ direction } \\
\hline \multirow[t]{2}{*}{$\begin{array}{l}\left(\mathrm{A}_{1}+\mathrm{A}_{3}\right),\left(\mathrm{A}_{2}+\mathrm{A}_{4}\right) \\
\left(\left(\mathrm{A}_{5}\right)\right) \\
\left(\mathrm{A}_{6}+\mathrm{A}_{7}\right) \\
\end{array}$} & \multicolumn{3}{|c|}{$\begin{array}{l}0 \text { diagonal; } 0 \text { non-diagonal } \\
4 \text { diagonal; } 3 \text { non-diagonal } \\
6 \text { diagonal; } 2 \text { non-diagonal }\end{array}$} \\
\hline & $\| z$ & $\| x$ & $y$ \\
\hline $\begin{array}{l}\left(\mathrm{X}_{1}+\mathrm{X}_{3}\right),\left(\mathrm{X}_{2}+\mathrm{X}_{4}\right) \\
\mathrm{X}_{5}\end{array}$ & $\begin{array}{c}0 \text { diagonal; } 0 \text { non-diagonal } \\
0\end{array}$ & $\begin{array}{c}2 \text { diagonal; } 2 \text { non-diagonal } \\
1\end{array}$ & $\begin{array}{c}0 \text { diagonal; } 0 \text { non-diagonal } \\
0\end{array}$ \\
\hline $\begin{array}{l}\left(X_{1}^{\prime}+X_{4}^{\prime}\right),\left(X_{2}^{\prime}+X_{3}^{\prime}\right) \\
X_{5}^{\prime}\end{array}$ & $\begin{array}{c}0 \text { diagonal; } 0 \text { non-diagonal } \\
0 \\
\end{array}$ & $\begin{array}{c}0 \text { diagonal; } 0 \text { non-diagonal } \\
0\end{array}$ & $\begin{array}{c}2 \text { diagonal; } 2 \text { non-diagonal } \\
1\end{array}$ \\
\hline $\begin{array}{l}\left(\mathrm{R}_{1}+\mathrm{R}_{4}\right),\left(\mathrm{R}_{2}+\mathrm{R}_{3}\right) \\
\left(\left(\mathrm{R}_{5}\right)\right)\end{array}$ & $\begin{array}{l}0 \text { diagonal; } 0 \text { non-diagonal } \\
4 \text { diagonal; } 1 \text { non-diagonal }\end{array}$ & $\begin{array}{l}0 \text { diagonal; } 0 \text { non-diagonal } \\
4 \text { diagonal; } 1 \text { non-diagonal }\end{array}$ & $\begin{array}{l}2 \text { diagonal; } 2 \text { non-diagonal } \\
4 \text { diagonal; } 2 \text { non-diagonal }\end{array}$ \\
\hline $\begin{array}{l}\left(\mathrm{R}_{1}^{\prime}+\mathrm{R}_{3}^{\prime}\right),\left(\mathrm{R}_{2}^{\prime}+\mathrm{R}_{4}^{\prime}\right) \\
\left(\left(\mathrm{R}_{5}^{\prime}\right)\right)\end{array}$ & $\begin{array}{l}0 \text { diagonal; } 0 \text { non-diagonal } \\
4 \text { diagonal; } 1 \text { non-diagonal }\end{array}$ & $\begin{array}{l}2 \text { diagonal; } 2 \text { non-diagonal } \\
4 \text { diagonal; } 2 \text { non-diagonal }\end{array}$ & $\begin{array}{l}0 \text { diagonal; } 0 \text { non-diagonal } \\
4 \text { diagonal; } 1 \text { non-diagonal }\end{array}$ \\
\hline
\end{tabular}


Table 3. Number of components of inverse effective mass tensor for different points in the Brillouin zone of crystals $\mathrm{ZnP}_{2}$ and $\mathrm{CdP}_{2}$.

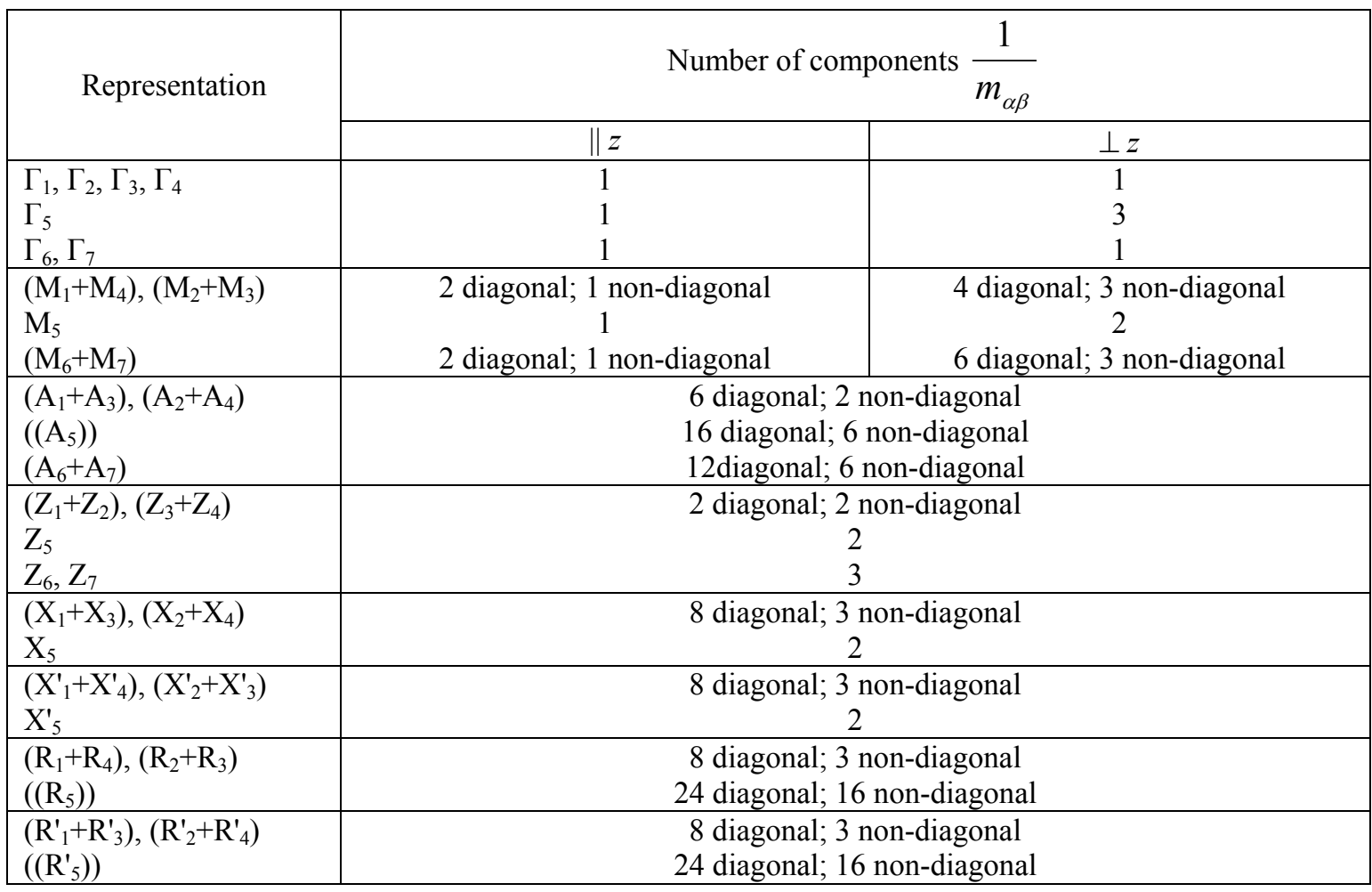

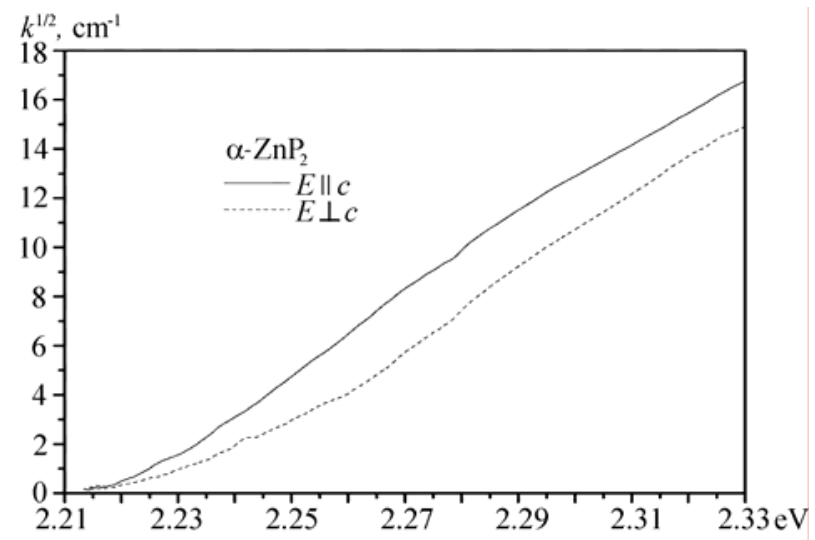

$$
k^{1 / 2}, \mathrm{~cm}^{-1}
$$

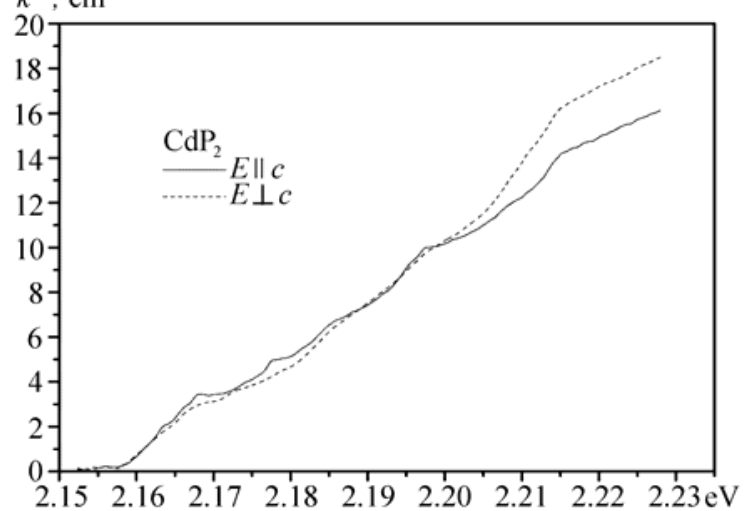

Fig. 4. Edge absorption spectra of tetragonal crystals $\mathrm{ZnP}_{2}$ and $\mathrm{CdP}_{2}$.
Comparing the spectra of exciton-phonon transitions, phonon Raman scattering $[1,2]$, and taking into consideration the results of our symmetry analysis (see Tables 1, 2, and 3) we can suppose that the minimum of conduction band may be located in $\mathrm{M}$ point. There are two considerations for this conclusion: 1) all $\Gamma-\mathrm{M}$ transitions are allowed in both polarizations, but some of $\Gamma-Z$ transitions are forbidden in $\vec{E} \| \mathrm{z}$ polarization (it was mentioned that the main feature of the obtained photoluminescence spectra (Fig. 2) is the equal number of bands with approximately the same phonon energies in both polarizations); 2) for electrons in $\mathrm{M}$ point, there are minimal number of non-zero components $d E\left(\vec{k}_{0}\right) / d k_{\alpha}$ in $\Gamma-\mathrm{M}$ direction and $\mathrm{M}$ point is the point of zero slope for all the dispersion curves of phonon states in the same direction. The points $\mathrm{Z}$ and $\mathrm{R}$ are not those of zero slope for all dispersion curves of electron states in the corresponding directions. In the point $\mathrm{A}$, there are nonzero components $d E\left(\vec{k}_{0}\right) / d k_{\alpha}$ both for electron and phonon states. Additional criteria to further selection of a candidate for the conduction band extremum can be obtained from the measurements of free-exciton luminescence in magnetic fields when the degeneration of energy states will be removed as caused by merging the states. For instance, the energy dispersion curves in $\Gamma-\mathrm{M}$ direction that corresponds to non-merged states $\mathrm{M}_{6}$ and $\mathrm{M}_{7}$ will have zero slope in $\mathrm{M}$ point. 
References

1. V.O. Gubanov, S.V. Koryakov, R.A. Poveda, Z.Z. Yanchuk, The analysis of dispersion of phonon states in tetragonal crystals $\mathrm{ZnP}_{2}$ and $\mathrm{CdP}_{2}$ by method of projective representation // Zhurn. Fiz. Doslidzhen' 6 (1), p. 109-117 (2002).

2. S. Garasevich, V. Gubanov, P. Korenyuk, S. Koryakov, A. Slobodyanyuk, Z. Yanchuk, Two- phonon Raman spectra of tetragonal crystals $\mathrm{ZnP}_{2}$, $\mathrm{CdP}_{2}$, and $\mathrm{TeO}_{2} / /$ SPIE Proc. 5507, p. 26 (2004).

3. V.O. Gubanov, S.V. Koryakov, Projective representations construction for different points of Brillouin zone. Application for space symmetry groups $\mathrm{P}_{4} 2_{1} 2$ and $\mathrm{P}_{3} 2_{1} 2 / /$ ArXiv: cond-mat. 0406519 (2004).

4. G.L. Bir, G.E. Pikus, Symmetry and deformation effects in semiconductors. Nauka, Moscow (1972). 